\title{
Isotope effect on hydrogen bond symmetrization in hydrogen and deuterium fluoride crystals by molecular dynamics simulation

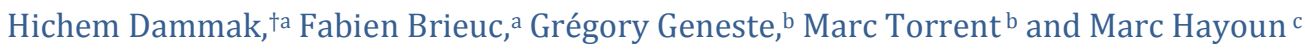

The isotope effect on the collective proton/deuteron transfer in hydrogen and deuterium fluoride crystals has been investigated at $100 \mathrm{~K}$ by ab initio quantumthermal-bath path-integral molecular dynamics (QTB-PIMD) simulation. The deuterons within a planar zigzag chain of the orthorhombic structure simultaneously flip between covalent and hydrogen bonds due to the barrier crossing through tunnelling. The height of the corresponding static barrier normalized for one deuteron is $29.2 \mathrm{meV}$. In the HF crystal, all the protons are located at the center of the heavyatom distance. This evidences the symmetrization of the H-bonds, and indicates that the proton zero-point energy is above the barrier top. The decrease of the heavy-atom distance due to quantum fluctuations in both HF and DF crystals corresponds to a large decrease and an increase of the hydrogen and covalent bond lengths, respectively. Upon deuteration, the increase of the heavy-atom distance (Ubbelohde effect) is in agreement with experimental data.

\section{Introduction}

Proton hopping through a hydrogen bond network has been evidenced in various hydrogen-bonded systems $^{1}$ such as liquid water ${ }^{2,3}$ and ordinary ice (hexagonal $\left.\mathrm{I}_{\mathrm{h}}\right)^{4}$ for example, but not in the hydrogen fluoride (HF) crystal. In fact, the orthorhombic structure of HF consisting of planar zigzag chains of HF molecules offers the possibility of collective multiple proton hopping through the formation and concomitant cleavage of covalent bonds involving neighbouring molecules.

The small mass of the proton means that it is inherently quantum mechanical in nature, and nuclear quantum effects (NQE) such as zero-point motion, quantum delocalization, and quantum tunnelling are relevant. The potential seen by the protons of the HF zigzag chain is a symmetric double well energy surface and the zero-point energy can be below or above the barrier top, thus defining two limit cases. i) The occupied proton energy levels are above the barrier top and the maximum of the position probability density is at the barrier top. This corresponds to a symmetrized hydrogen bond and there is then no proton hopping. ii) The occupied proton energy levels are far below the barrier top and the proton transfer mostly corresponds to (non- adiabatic) tunnelling. Proton tunnelling represents the motion of protons through the potential energy barrier that separates the final from the initial state when the thermally activated process of hopping over the barrier top is classically not possible. Proton tunnelling gets strongly enhanced upon decreasing the height and/or the width of the energy barrier. It typically requires short hydrogen bonds since the strength of hydrogen bonds is a key feature influencing the proton tunnelling. ${ }^{5}$ Among the hydrogen halides, HF is precisely the one having the strongest hydrogen bonds due to the largest electronegativity of fluorine and hence provides a rich playground for the study of the impact of NQE on proton position disorder.

We report herein a simulation study in which we investigate the impact of $\mathrm{NQE}$ on the $\mathrm{HF}$ and $\mathrm{DF}$ crystals by using the quantum thermal bath pathintegral molecular dynamics (QTB-PIMD) simulation technique that efficiently takes into account the NQE. The interactions were modelled from first principles within the framework of density functional theory (DFT). The simulated system and the QTB-PIMD method are described in section 2 . The results on the proton disorder, including the static energy profile, the position probability density, and the partial radial 
distribution functions are given in section 3. Sections 4 and 5 are dedicated to the discussion and the conclusion, respectively.

\section{System under study and methods}

\subsection{Simulated System}

The structure of our simulated crystal is composed of two zigzag HF chains as shown in Fig. 1. Each $\mathrm{H}$ atom is bonded by a covalent bond to its first neighbour and by a hydrogen bond to its second neighbour. The protons within a chain could simultaneously flip between the covalent and the hydrogen bonds.

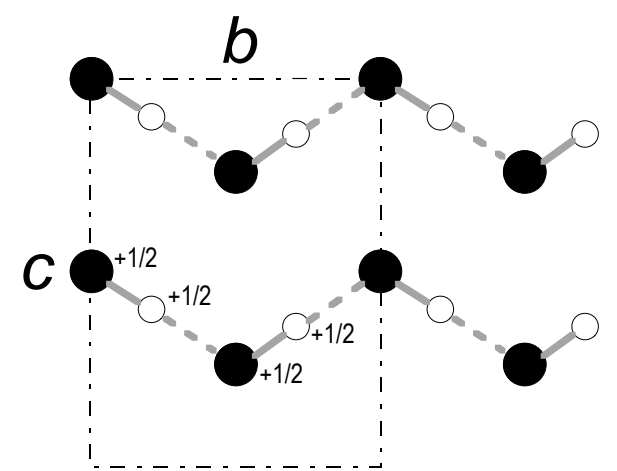

Fig. 1 Simulated HF crystal (8 molecules) in the $B m 2{ }_{1} b$ structure. $\mathrm{F}$ and $\mathrm{H}$ atoms are black and white circles, respectively. Covalent and hydrogen bonds are also displayed. The orthorhombic primitive cell is indicated by the black rectangle whose lattice parameters $b$ and $c$ are shown. The $a$ axis is pointing into the page. The first chain is in the plane at $x=0$ while the second one is located in the plane at $x=\frac{1}{2}$.

It is useful to consider the approximate onedimensional double-well model, in which all the protons in one of the two chains are constrained at intermediate positions between their two neighbouring $\mathrm{F}$ atoms. The corresponding reaction coordinate was chosen as the difference between the distance of the protons from their neighbouring $\mathrm{F}$ atoms:

$\xi=d\left(\mathrm{H}-\mathrm{F}^{(2)}\right)-d\left(\mathrm{H}-\mathrm{F}^{(1)}\right)$

When $\xi=0$, the protons of one chain are exactly in the center of the F-F distance.

\subsection{Quantum Thermal Bath Path-Integral Molecular} Dynamics Simulation

NQE cannot be neglected in HF and DF and can be accounted for by using the path-integral (PI) formalism.6,7 In this formulation of quantum statistical mechanics, the canonical partition function
$Z$ is written as a discretized imaginary time path integral. For a quantum system containing $N$ (distinguishable) particles of mass $m, Z$ can be expressed according to:

$$
\begin{aligned}
& Z=\lim _{P \rightarrow \infty}\left(\frac{2 \pi m P k_{B} T}{h^{2}}\right)^{\frac{3 N P}{2}} \times \\
& \int e^{-\beta V_{e f f}\left(\left\{\mathbf{r}^{N}\right\}^{(1)}, \ldots,\left\{\mathbf{r}^{N}\right\}^{(P)}\right)}\left\{d \mathbf{r}^{N}\right\}^{(1)} \ldots\left\{d \mathbf{r}^{N}\right\}^{(P)}
\end{aligned}
$$

The integral is over $P$ (Trotter number) replicas of the system, labelled by the integer $s$, each replica being a set of $N$ positions of the atoms $\left\{\mathbf{r}^{N}\right\}^{(s)}=\left(\mathbf{r}_{1}^{(s)}, \ldots, \mathbf{r}_{N}^{(s)}\right)$. These replicas come from the discretization of the PI in imaginary time. The effective potential $V_{\text {eff }}$ which depends on all atomic positions of all replicas is composed of two terms, the physical potential energy, $V$, computed in each replica and averaged over them, and a harmonic coupling term, of angular frequency $\omega_{P}=\sqrt{P} / \beta \hbar$, between replicas:

$V_{e f f}\left(\left\{\mathbf{r}^{N}\right\}^{(1)}, \ldots,\left\{\mathbf{r}^{N}\right\}^{(P)}\right)=\sum_{s=1}^{P}\left[\frac{1}{P} V\left(\left\{\mathbf{r}^{N}\right\}^{(s)}\right)+\right.$ $\left.\sum_{i=1}^{N} \frac{1}{2} m \omega_{P}^{2}\left(\mathbf{r}_{i}^{(s)}-\mathbf{r}_{i}^{(s+1)}\right)^{2}\right]$

This effective potential has the particularity to depend on the atomic masses and on the temperature. Each particle $i$ of the replica $s$ is thus interacting through harmonic forces with the particles $i$ of the replicas $(s+1)$ and $(s-1)$, forming a polymer ring that closes on itself by periodic boundary conditions, $\mathbf{r}_{i}^{(P+1)}=\mathbf{r}_{i}^{(1)}$. In the limit where the Trotter number $P \rightarrow$ $\infty$, this equivalent classical system has the same partition function as that of the quantum system. As a consequence, MD simulation can be applied to the classical equivalent to numerically estimate the static properties of the quantum system. For a PIMD simulation in the microcanonical ensemble, the corresponding equation of motion of each particle $i$ in each replica $s$ writes

$m \ddot{\mathbf{r}}_{i}^{(s)}=-\frac{1}{P} \nabla_{\mathbf{r}_{i}^{(s)}} V\left(\left\{\mathbf{r}^{N}\right\}^{(s)}\right)-m \omega_{P}^{2}\left(2 \mathbf{r}_{i}^{(s)}-\mathbf{r}_{i}^{(s+1)}-\mathbf{r}_{i}^{(s-1)}\right)$

In practical PIMD simulations, the Trotter number is finite, and must be chosen to converge the estimated quantities. For instance, the average total energy of the system is given by the following estimator: 
Phys. Chem. Chem. Phys. 21 (2019) pp.3211-3217

$\langle E\rangle=\left\langle\sum_{s=1}^{P} \sum_{i=1}^{N} \frac{\left(\mathbf{p}_{i}^{(s)}\right)^{2}}{2 m}-\sum_{s=1}^{P} \sum_{i=1}^{N} \frac{1}{2} m \omega_{P}^{2}\left(\mathbf{r}_{i}^{(s)}-\mathbf{r}_{i}^{(s+1)}\right)^{2}\right\rangle+$ $\left\langle\sum_{s=1}^{P} \frac{1}{P} V\left(\left\{\mathbf{r}^{N}\right\}^{(s)}\right)\right\rangle$

where the first and second averages are the kinetic and the potential energies, respectively. This primitive estimator can be derived from $-\partial \operatorname{Ln}(Z) / \partial \beta$. Exact results can be obtained by PIMD simulations but often at the price of a high computational cost.

The quantum thermal bath (QTB) $\mathrm{MD}^{8}$ is an approximate alternative method including the quantum fluctuations, and is based on a modification of the Langevin thermostat. ${ }^{9}$ The cartesian component $\alpha$ of the random force, $R_{i \alpha}$, applied on the atom $i$, is not a white noise and its power spectral density, $I_{R}$, is derived from the quantum dissipationfluctuation theorem, ${ }^{10}$ and is related to the Fourier transform of the autocorrelation function, $\left\langle R_{i \alpha}(t) R_{i \alpha}(t+\tau)\right\rangle$, according to the Wiener-Khinchin theorem:

$\left\langle R_{i \alpha}(t) R_{i \alpha}(t+\tau)\right\rangle=\int_{-\infty}^{+\infty} I_{R}(\omega, T) \exp (-i \omega \tau) \frac{\mathrm{d} \omega}{2 \pi}$

$I_{R}(\omega, T)=2 m \gamma \theta(\omega, T)$

where $\gamma$ is the frictional coefficient. $\theta(\omega, T)$ is the average energy of the harmonic oscillator:

$\theta(\omega, T)=\hbar \omega\left[\frac{1}{2}+\frac{1}{\exp (\beta \hbar \omega)-1}\right]$

where $\omega$ is the angular frequency of the oscillator, $\hbar$ is the reduced Planck constant and $\beta$ the statistical temperature $\left(1 / k_{B} T\right)$. The equation of motion is thus:

$m \ddot{\mathbf{r}}_{i}=-\nabla_{\mathbf{r}_{i}} V\left(\left\{\mathbf{r}^{N}\right\}\right)-m \gamma \dot{\mathbf{r}}_{i}+\mathbf{R}_{i}$

In contrast to the Langevin thermostat, $I_{R}$ is $\omega$ dependent and the random force components are generated using the procedure detailed in Ref. 11 and 12). The QTB method provides exact results in the case of purely harmonic systems. For anharmonic systems and as for all methods based on classical trajectories, ${ }^{13}$ QTB-MD can fail due to zero-point energy leakage (ZPEL), which is the consequence of the coupling between vibrational modes. In this case, the resulting energy distribution does not match Eq. (8), it is intermediate between the quantum distribution and the classical homogeneous distribution. Weakly anharmonic systems can be successfully simulated by QTB-MD by increasing the value of $\gamma$, whereas for strongly anharmonic systems the ZPEL cannot be suppressed and QTB-MD should not be used.

It is thus suitable to combine the QTB and PIMD, ${ }^{14}$ in a way similar to that developed by Ceriotti et al. ${ }^{15}$ in order to improve the convergence of the PIMD and/or to correct potential failures of the QTB-MD technique especially in the case of strongly anharmonic systems. This combination requires the modification of the power spectral density of the random forces applied on each atom of each replica. Indeed, for not converged Trotter number, quantum fluctuations are already partially included within the ring polymer of the PIMD. The QTB random forces will thus only bring the missing part of the NQE which is dependent on the Trotter number. In practice, $\theta(\omega, T)$ in Eq. (7) is replaced by the adequate function $\kappa_{P}(\omega, T)$, which is solution of the following equation

$\frac{1}{P} \frac{k_{B} T}{m \omega_{k=0}^{2}}+\frac{1}{P} \sum_{k=1}^{P-1} \frac{\kappa_{P}\left(\omega_{k}, T\right)}{m \omega_{k}^{2}}=\frac{\theta(\omega, T)}{m \omega^{2}}$

where $\omega_{k}$ is the angular frequency of the normal modes of the ring polymer in the harmonic approximation:

$\omega_{k}^{2}=\frac{\omega^{2}}{P}+4 \omega_{P}^{2} \sin ^{2}\left(\frac{k \pi}{P}\right)$

For the normal modes at $k>0$, the power spectral density is thus given by:

$I_{R}(\omega, T)=2 m \gamma \kappa_{P}(\omega, T)$

whereas the normal mode at $k=0$ (centroid of the ring polymer) is classically considered $\left(2 m \gamma k_{B} T\right)$. Since the corresponding random forces are intended to be applied to the normal modes, the random forces applied on the atoms are obtained through an orthogonal transformation which can be found in Ref. 14.

\subsection{Computational details}

The proton/deuteron transfers require the quantum mechanical description of the electronic structure that allows for the breaking and the formation of the chemical and hydrogen bonds. This description is achieved by using density-functional theory (DFT) calculations within the GGA-PBE functional. ${ }^{16} \mathrm{We}$ employed the ABINIT code ${ }^{17}$ and our simulations are performed in the framework of the projector 
augmented-wave (PAW) method. ${ }^{18-20}$ We used, in all the MD simulations, an 8-molecules supercell consisting of $1 \times 2 \times 1$ orthorhombic cells of $4 \mathrm{HF}$ (DF) molecules, containing two zig-zag chains along the $y$ direction. The Brillouin zone of this supercell is sampled with a $4 \times 2 \times 3$ Monkhorst-pack k-point mesh, and the plane-wave cut-off is set to 30 hartrees; in the augmentation regions it is set to 60 hartrees for the density. The lattice constants of the supercell are maintained fixed in all the MD runs, based on the values found by structural optimization of the primitive cell, i.e. $a=4.080 \AA, \quad b=4.062 \AA$ and $c=5.615 \AA$.

The QTB-PIMD technique, we implemented in the parallel version of the ABINIT code, 17,19 was used to simulate the proton (deuteron) transfers in HF (DF) with a Trotter number $P=56$, for which the calculations were converged. This simulation provides a description based on the atomic-position probability density at equilibrium and cannot give any information about the kinetics of the process. The simulations were carried out, with the static lattice constants, at $T=100 \mathrm{~K}$ in the canonical (NVT) ensemble through the QTB thermostat. The nuclear masses were taken at 1 amu for $\mathrm{H}, 2$ amu for D and 19 amu for $\mathrm{F}$. The time step and the effective frictional coefficient were set at $\delta t=0.726$ fs and $\gamma=2 \mathrm{THz}$. The systems were equilibrated for about 2-3 ps, and once equilibrated, averages were computed along runs of about $25 \mathrm{ps}$ and $74 \mathrm{ps}$ as long for $\mathrm{HF}$ and $\mathrm{DF}$, respectively.

\section{Results}

\subsection{Static calculation}

The first step of the investigation was the static calculation of the collective proton transfer. It consists in the determination at $T=0 \mathrm{~K}$ of the free energy along the reaction coordinate given by Eq. (1). The protons within one chain simultaneously flip between the covalent and the hydrogen bonds.

We computed the static energy profile, that is experienced by the protons of one chain, by using the Nudged Elastic Band method ${ }^{21}$ implemented in the ABINIT code. The result thus obtained is shown by the open circles in Fig. 2. This symmetric double-well energy profile can be expressed through the relation:

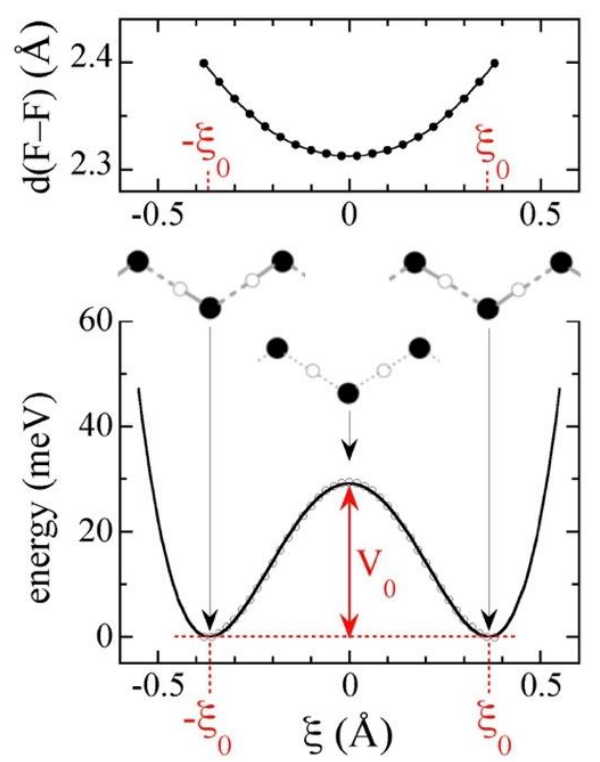

Fig. 2 Double-well energy profile normalized for one proton as a function of the reaction coordinate, $\xi$ (Eq. (1)). Open circles: static free energy computed by the Nudged Elastic Band method implemented in ABINIT; solid line: result of the fitting on the open circles of the parameters $V_{0}$ and $\xi_{0}$ of $V(\xi)$ in Eq. (2). The evolution of the distance between the first and second neighbours of the proton, $d(\mathrm{~F}-\mathrm{F})$, is also displayed as a function of $\xi$.

$V(\xi)=V_{0}\left[1-\left(\xi / \xi_{0}\right)^{2}\right]^{2}$

The values of the two parameters $V_{0}$ and $\xi_{0}$ have been fitted on the calculated energy profile. The height of the barrier is $V_{0}=29.2 \mathrm{meV}$ and the distance between the two wells is $2 \xi_{0}=0.73 \AA$. Fig. 2 also displays the atomic configurations associated to the two energy minima, $\xi= \pm \xi_{0}$, and to the saddle point, $\xi=0$. Moreover, during the transfer, the distance between the first and second neighbours of the proton, $d(F-$ $F)$, varies and reaches a minimum value at $\xi=0$. The amplitude of variation of this distance is $0.077 \AA$. Such a correlation between the two coordinates, $\xi$ and $d(F-F)$, is clearly evidenced by the two-dimensional energy surface shown in Fig. 3.

\subsection{Probability density for collective proton transfer}

The probability densities, $\rho$, computed by QTB-PIMD for the proton/deuteron in HF/DF along the reaction coordinate, $\xi$, are given in Fig. 4 . The probability density for DF (blue distribution) displays two maxima. This indicates that the deuterons flip 
Phys. Chem. Chem. Phys. 21 (2019) pp.3211-3217 between two stable positions due to the barrier crossing through the tunnel effect. This effect is important as shown by the value of $\rho=0.61 \AA^{-1}$ at $\xi=$ 0 . Without this effect, i.e. in the classical regime, the system would remain in one of the two potential energy minima as obtained by standard MD (grey peak). Another significant impact of quantum effects is the negative shift of the stable position by about $-0.1 \AA$. In the case of HF (red distribution), the only most probable position is around $\xi=0$, meaning that the protons are located at mid-distance between their two first fluoride neighbours. Hence, the image of protons linked to the two fluoride ions by a covalent bond and a H-bond probably no longer holds. In fact, protons are linked to the two fluoride ions by two equivalent bonds. It is a case of symmetrization of the hydrogen bond 22 occurring when the proton zeropoint energy is above the barrier top. ${ }^{23}$ The comparison of the probability densities of HF and DF clearly evidences an important isotope effect.

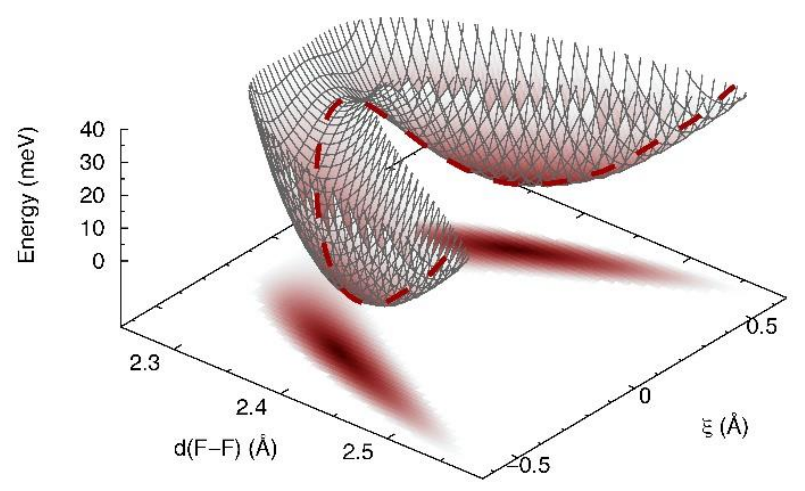

Fig. 3 Energy surface normalized for one proton as a function of the reaction coordinate $\xi$ and $d(\mathrm{~F}-\mathrm{F})$, as obtained by a static calculation without any relaxation of the atomic positions on the $B m 2_{1} b$ structure. The energy path of Fig. 2 is displayed by the red dashed line.

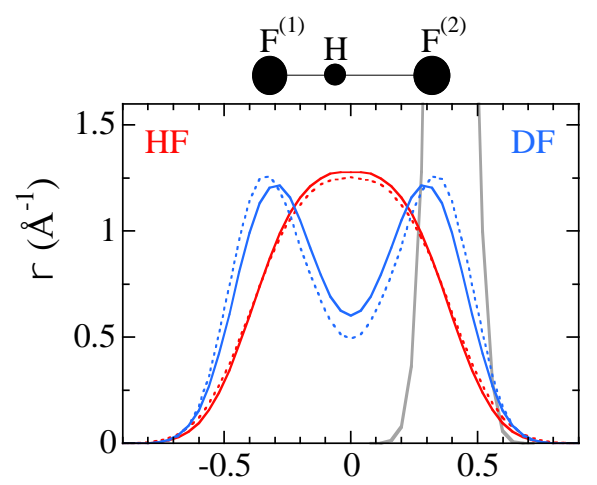

$(\AA)$
Fig. 4 Probability density, $\rho$, at $T=100 \mathrm{~K}$ for the proton in $\mathrm{HF}$ (red lines) or deuteron in DF (blue lines) as a function of the reaction coordinate $\xi$ (Eq. (1)). Solid lines: QTB-PIMD, $P=56$; red dashed-line: PIMD, $P=128$; blue dashed-line: QTB-MD, $\gamma=4 \times 10^{-3}$ a.u. The light-grey line is the probability density as obtained by standard MD without NQE.

Note that the PIMD probability density for HF, $\rho$, requires 128 replicas (red dashed-line) to approach the converged QTB-PIMD result, $\rho_{0}$. The discrepancy between the two curves can be evaluated by calculating the divergence factor, $d_{\rho}$ :

$d_{\rho}=\left[\frac{\int\left(\rho-\rho_{0}\right)^{2} d \xi}{\int \rho_{0}^{2} d \xi}\right]^{1 / 2}$

which is similar to the reliability factor used in the Rietveld method. ${ }^{24}$ Its value is $d_{\rho} \approx 3 \%$. In addition, QTB-MD with a very high value of the friction coefficient provides an approximate distribution for DF (blue dashed-line) which is qualitatively similar to the QTB-PIMD probability density but the tunnelling effect is underestimated. The divergence factor with respect to the QTB-PIMD result is $d_{\rho} \approx 12 \%$.

The simulated probability density along the reaction coordinate, $\xi$, using the centroid positions at $T=100 \mathrm{~K}$ can be used to determine the effective free energy profiles $^{25}$ for proton/deuteron. These profiles are displayed in Fig. 5 as a function of the reaction coordinate, $\xi$. The effective barrier height is lowered through tunnelling for DF (blue line). Its decrease is about $40 \%$ with respect to the static barrier height (29.2 meV). For HF (red line) the proton behaves as if there was no barrier, indicating that the proton zeropoint energy is above the barrier top.

\subsection{Radial distribution functions}

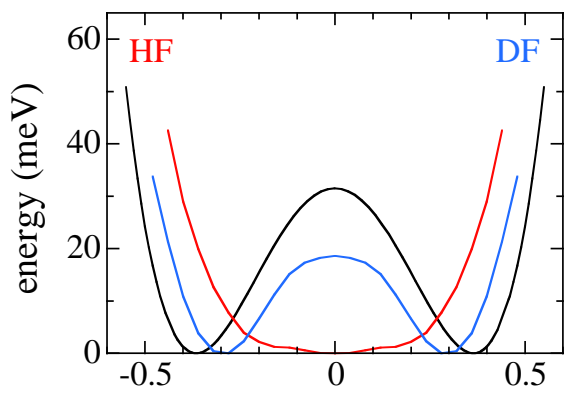

$(\AA)$

Fig. 5 Energy profiles normalized for one proton/deuteron as a function of the reaction coordinate, $\xi$ (Eq. 1). Simulated free energy profiles at $T=100 \mathrm{~K}$ in HF (red line) and DF (blue line) deduced from the probability densities, $\rho_{c}$, using the centroid 
positions (QTB-PIMD, $P=56$ ) through the expression $-k_{B} T \ln \left(\rho_{c} / \rho_{\max }\right)$, where $\rho_{\max }$ is the maximum value of the probability density. Black line: static double-well energy, $V(\xi)$, of Fig. 2.

Fig. 6 displays the radial distribution functions, $g(r)$, obtained from QTB-PIMD trajectories at $T=100 \mathrm{~K}$. The H-F and D-F $g(r)$ show peaks in the 0.8-1.6 range. For DF (blue curve of Fig. $6 a$ ), the first peak at $1.04 \AA$ and the second peak at $1.33 \AA$ correspond to the covalent and hydrogen bonds, respectively. In comparison to the classical case (grey curve of Fig. 6a), the covalent bond length is slightly enlarged while that of the H-bond is greatly shrinked. Indeed, quantum fluctuations probe the anharmonic region of the potential energy and are more relevant for shallow energy profiles. The hydrogen bond is weaker than the covalent one and therefore more sensitive to quantum effects. This quantum effect is more pronounced in the crystal with the lighter isotope (red curve of Fig. 6a) and leads to a single peak at $1.1 \AA$, which corresponds to a symmetrization of the hydrogen bond. Note also that the increase of the hydrogen bond length with deuteration is known as the Ubbelohde effect. ${ }^{26}$

The $g(r)$ of Fig. $6 \mathrm{~b}$ indicates that the F-F distance between the first and second neighbours of $\mathrm{H}$ $\left(2.34_{6} \AA\right)$ and $D\left(2.37_{1} \AA\right)$ is shortened in both crystals due to quantum fluctuations. The overall decrease of the F-H-F length corresponds to a large decrease of the H-bond length and an increase of the length of the covalent bond. The discrepancy with respect to the MD result (grey curve) is about $-0.05_{4} \AA$ and $-0.03_{3} \AA$ for $\mathrm{HF}$ and $\mathrm{DF}$, respectively. In the case of $\mathrm{HF}$, our value is larger than the one $\left(-0.03_{5} \AA\right)$ found by Li et $a{ }^{5}$ showing that their PIMD calculation was not fully converged, since they used a too small number of replicas $(P=16)$. Moreover, our PIMD calculations showed that $P=16$ is not sufficient to have the H-bond symmetrization, and provides a probability density with two maxima. On the other hand, these authors point out the correct effect, i.e. that in strong $\mathrm{H}$ bonded systems, as HF, NQE result in shorter $\mathrm{H}$ bonds.
The effect of deuteration on the F-F distance has been estimated from neutron powder diffraction on DF at $T=85 \mathrm{~K}$ by Johnson et al. ${ }^{27}(2.51 \pm 0.02 \AA)$ and from Xray diffraction on $\mathrm{HF}$ at $T=148 \mathrm{~K}$ by Atoji et al. ${ }^{28}$ $(2.49 \pm 0.01 \AA)$. It results in an elongation of the F-F distance of about $0.02 \AA$ which is satisfactorily reproduced by our QTB-PIMD simulations, $0.02{ }_{5} \AA$, at $T=100 \mathrm{~K}$.

In contrast, the $g(r)$ for $\mathrm{H}-\mathrm{H}$ and D-D of Fig. 6c display a rather small impact of quantum effects on the first peak, resulting in a slight shift $(-0.03 \AA)$ and widening with respect to the classical case.

\subsection{Crystal structure}

The centroid position distributions of the protons/deuterons in the $b c$ plane at $T=100 \mathrm{~K}$ in HF/DF are displayed in Fig. 7. Here, all the protons/deuterons positions are explicitly shown. In $\mathrm{HF}$, the symmetrization clearly occurs for all the hydrogen bonds leading to the $B m m b$ space group symmetry. In contrast, all the deuterons in DF occupy two favoured positions, in between their two neighbours, to which they are bonded either by a covalent bond or a H-bond. Moreover, one can note that the zigzag chain structure is antiparallel with respect to the orientations of the covalent bonds. This corresponds to the Pmnb space group symmetry. It is important to mention that the detailed analysis of the simulation trajectory shows that only $65 \%$ of the configurations are in the antiparallel structure. Moreover, within the statistical accuracy of the computation, the antiparallel and parallel structures could have the same probability. We note, that although our conclusion on the antiparallel chain structure could be correct, it is the opposite of the parallel-chain model considered to represent the structure in the neutron powder diffraction study on DF at $T=85 \mathrm{~K}$ by Johnson et al. ${ }^{27}$

\section{Discussion}

Among the possible space groups, Atoji et al. ${ }^{28}$ proposed the $B m m b$ structure for $\mathrm{HF}$ at $T=148 \mathrm{~K}$ without any experimental evidence for the location of the protons, since they used the X-ray diffraction method. In this space group, the authors suggested 
Phys. Chem. Chem. Phys. 21 (2019) pp.3211-3217 that the protons occupy either of two equally probable positions on both sides of the centre of $\mathrm{F}-\mathrm{H}-$ F. However, according to this symmetry the protons can occupy the centre of $\mathrm{F}-\mathrm{H}-\mathrm{F}$, corresponding to the symmetrization of the hydrogen bond we observed (Fig. 7). The important broadening of the infrared absorption bands of HF compared to those of other hydrogen halide crystals ( $\mathrm{HI}, \mathrm{HBr}$ and $\mathrm{HCl}$ ) evidenced the presence of very strong hydrogen bonds. ${ }^{29}$
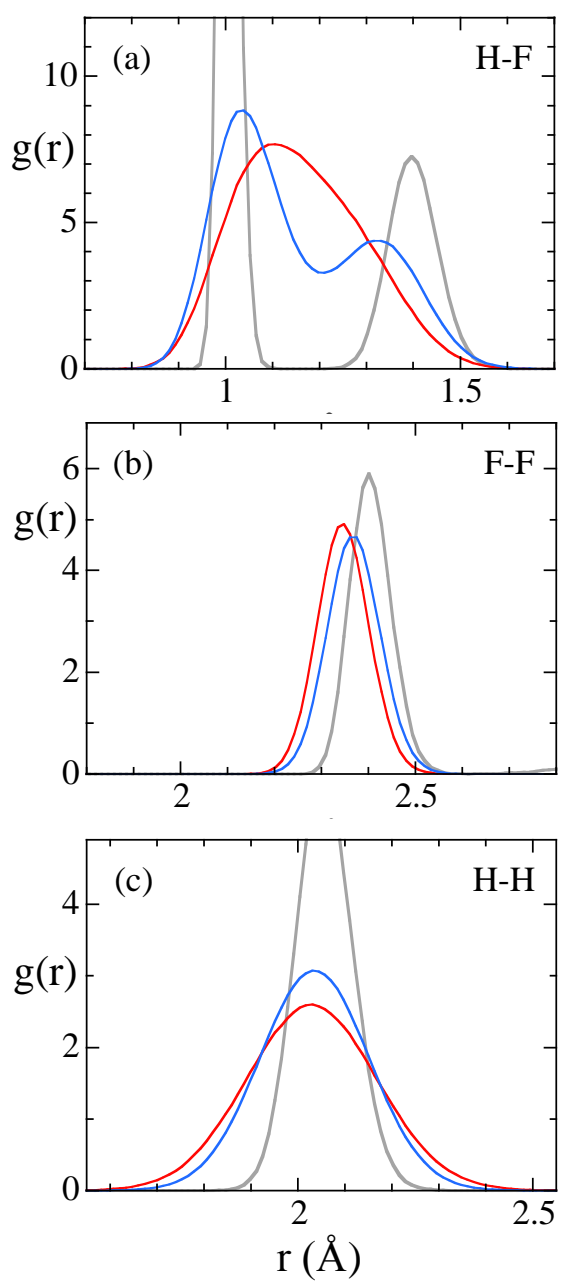

Fig. 6 Partial radial distribution functions, $g(r)$, computed at $T=$ $100 \mathrm{~K}$ in HF (red lines) and DF (blue lines) by QTB-PIMD $(P=56)$ compared to standard MD (light grey). (a) H-F (D-F), (b) F-F, (c) H-H (D-D). The same scale is used for the abscissa axes.

This experimental observation could be the consequence of quantum fluctuations of the proton feeling a strongly anharmonic potential energy similar to the red energy profile of Fig. 5. Nevertheless, the experimental evidence of the symmetrization of the H-bond in HF by Raman spectroscopy ${ }^{30}$ was found at pressures higher than $6 \mathrm{GPa}$, whereas our computation was carried out at a lower pressure. Indeed, the pressure along $\mathbf{b}$, which is the relevant axis for the zigzag chain structure is about $-1 \mathrm{GPa}$. It is worth noting that the zigzag structure of the chains allows the free variation of the bond lengths, since the F-H-F-H-F angles (in the bc plane) can accommodate the constraint induced by the fixed size of the box along $\mathbf{b}$. The pressure shift from the experimental value can be interpreted by a proton transfer barrier height too low due to the use of the PBE functional. Indeed, standard GGA functionals are usually thought as underestimating proton transfer barriers, at least for molecules in the gas phase $\mathrm{e}^{31}$ and to a lesser extent for single proton transfers in some bulk materials. ${ }^{23,32}$ This underestimation is attributed to a self-interaction error. ${ }^{33}$ In water, the PBE functional can also reverse the sign of the isotope fractionation between liquid and vapour through an important softening of the covalent bond. ${ }^{34}$ However, recent works ${ }^{35-37}$ moderate this trend since in some 2D or 3D hydrogen bond networks, proton transfer barriers as described by PBE are in good agreement with barriers obtained by more sophisticated functionals such as PBE0 or HSE. In the present 1D hydrogen bond network, a slight underestimation of the proton transfer barrier height may thus be expected, in relation with a slightly too strong hydrogen bond, which is reflected in the underestimation of the $b$ lattice constant by $5 \%$ within PBE. In addition, Zhang et al. ${ }^{38}$ found the symmetrization of the $\mathrm{H}$-bond at $25 \mathrm{GPa}$ within a static calculation by DFT at $T=0 \mathrm{~K}$. They claim an excellent agreement with experiment ${ }^{30}$ despite the absence of any NQE. This agreement probably results from the fact that the error of PBE on the transfer barrier would be cancelled by the absence of NQE in the calculation.

In the case of DF, no symmetrization of the H-bond is found neither in our computation (pressure along $\mathbf{b}$ of $-0.6 \mathrm{GPa}$ ) nor by experimental Raman spectroscopy at pressures up to $12 \mathrm{GPa}^{30}$

\section{Conclusions}

NQE in the HF and DF crystals have been investigated by QTB-PIMD simulation at $T=100 \mathrm{~K}$. An important isotope effect on the proton position disorder has been observed. 
We have evidenced a collective deuteron transfer in the zigzag chains of molecules located in the $(a, b)$ plane of the orthorhombic structure. The deuterons within a chain simultaneously flip between covalent and hydrogen bonds. Correlatively, the distances between their two first fluoride neighbours vary and reach a minimum value at the saddle point. The height of the associated static barrier normalized for one deuteron is $29.2 \mathrm{meV}$ between the two wells corresponding to a deuteron hopping length of about $0.36 \AA$. The simulated probability density for DF indicates that the deuterons cross the barrier through tunnelling. The effective barrier height is then $19 \mathrm{meV}$ and the associated hopping length is $0.29 \AA$. In addition, with a probability of $65 \%$ the zigzag chain structure in DF was found to be antiparallel with respect to the orientations of the covalent bonds.
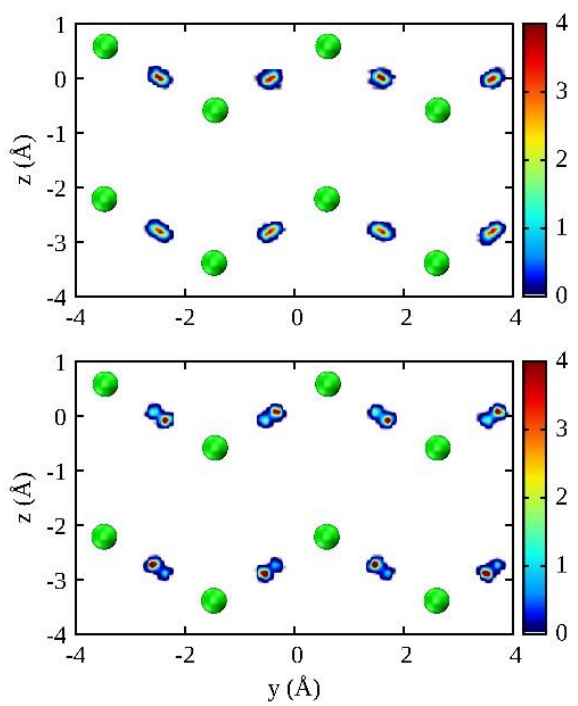

Fig. 7 Centroid position distributions of all protons/deuterons in the crystallographic $b c$ plane at $T=100 \mathrm{~K}$ in HF (upper part) and DF (lower part), as obtained from equilibrium QTB-PIMD trajectories. The mean positions of the $\mathrm{F}$ atoms are shown by the green spheres.

In the case of HF, the protons are located in the centre of the F-F distance resulting in a symmetrization of all the hydrogen bonds. This indicates that the proton zero-point energy is above the barrier top. As expected, the NQE are more pronounced in the HF crystal, owing to the smaller mass of hydrogen.

Finally, the overall decrease of the shortest heavyatom distance in both crystals consists especially in a large decrease of the H-bond length. In addition, the deuteration results in the (conventional) Ubbelohde effect which corresponds to an increase of the hydrogen bond length and an elongation of the F-F distance.

\section{Acknowledgements}

This work was performed using HPC resources from GENCI/CINES under 2016 grant C2016097631 and 2017 grant A0020907631. Part of this work was also performed using HPC resources from the "Mésocentre" computing centre of CentraleSupélec and École Normale Supérieure Paris-Saclay supported by CNRS and Région Île-de-France (http://mesocentre.centralesupelec.fr/) and from École Polytechnique through the LLR-LSI project.

\section{References}

1 D. Marx, Chem. Phys. Chem., 2006, 7, 1848.

2 A. Hassanali, F. Giberti, J. Cuny, T.D. Kühne and M. Parrinello, Proc. Natl. Acad. Sci. USA, 2013, 110, 13723.

3 E. Codorniu-Hernández and P.G. Kusalik, Proc. Natl. Acad. Sci. USA, 2013, 110, 13697.

4 C. Drechsel-Grau and D. Marx, Phys. Chem. Chem. Phys., 2017, 19, 2623.

5 X.-Z. Li, B. Walker and A. Michaelides, Proc. Natl. Acad. Sci. USA, 2011, 108, 6369.

6 M. E. Tuckerman, in Statistical Mechanics: Theory and Molecular simulation, Oxford University Press, New York, 2010, p. 475.

7 D. Marx, M. E. Tuckerman, J. Hutter and M. Parrinello, Nature: London, 1999, 397, 601.

8 H. Dammak, Y. Chalopin, M. Laroche, M. Hayoun and J.J. Greffet, Phys. Rev. Lett., 2009, 103, 190601.

9 N. Binggeli and J.R. Chelikowsky, Phys. Rev. B, 1994, 50, 11764.

10 H. B. Callen and T. A. Welton, Phys. Rev., 1951, 83, 34.

11 Y. Chalopin, H. Dammak, M. Laroche, M. Hayoun and J.J. Greffet, Phys. Rev. B, 2011, 84, 224301.

12 F. Brieuc, Y. Bronstein, H. Dammak, P. Depondt, F. Finocchi, and M. Hayoun, J. Chem. Theory Comput., 2016, 12, 5688.

13 M. Ben-Nun, R. D. Levine, J. Chem. Phys., 1994, 101, 8768.

14 F. Brieuc, H. Dammak and M. Hayoun, J. Chem. Theory Comput., 2016, 12, 1351.

15 M. Ceriotti, D. E. Manolopoulos and M. Parrinello, J. Chem. Phys., 2011, 134, 084104.

16 J. P. Perdew, K. Burke and K. Ernzerhof, Phys. Rev. Lett., 1996, 77, 3865.

17 X. Gonze et al., Computer Phys. Commun., 2009, 180, 2582.

18 P. Blöchl, Phys. Rev. B, 1994, 50, 17953.

19 M. Torrent, F. Jollet, F. Bottin, G. Zerah, and X. Gonze, Comput. Mater. Sci., 2008, 42, 337.

20 F. Jollet, M. Torrent and N. Holzwarth, Comput. Phys. Commun., 2014, 185, 1246.

21 D. Sheppard, R. Terrell, and G. Henkelman, J. Chem. Phys., 2008, 128, 134106

22 M. Benoit, D. Marx, and M. Parrinello, Nature, 1998, 392, 258.

23 G. Geneste, Solid State Ionics, 2018, 323, 172. 
Phys. Chem. Chem. Phys. 21 (2019) pp.3211-3217

24 R. A. Young, Ed., The Rietveld Method, Oxford University Press, Oxford, U.K., 1993.

25 D. Laria, G. Ciccotti, M. Ferrario, and R. Kapral, Chem. Phys., 1994, 180, 181.

26 A. R. Ubbelohde and K. J. Gallagher, Acta Cryst., 1955, 8, 71.

27 M. W. Johnson, E. Sandor and E. Arzi, Acta Cryst. B, 1975, 31, 1998.

28 M. Atoji and W. N. Lipscomb, Acta Cryst. 1954, 7, 173.

29 P. A. Giguère, and N. Zengin, Can. J. Chem., 1958, 36, 10019.

30 D. A. Pinnick, A. I. Katz and R. C. Hanson, Phys. Rev. B, 1989, 39, 8677.

31 V. Barone and C. Adamo, J. Chem. Phys., 1996, 105, 11007.

32 See the supplementary material in Y. Shi, J. Qi, Y. Han and T. Lu, Phys. Rev. Appl., 2018, 10, 024021.

33 See the supplementary material in Ref. 35.

34 L. L. Wang, M. Ceriotti and T. E. Markland, J. Chem. Phys., 2014, 141, 104502; O. Marsalek and T. E. Markland, J. Phys. Chem. Lett., 2017, 8, 1545.

35 X.-Z. Li, M. I. J. Probert, A. Alavi and A. Michaelides, Phys. Rev. Lett., 2010, 104, 066102.

36 J. Chen, X.-Z. Li, Q. Zhang, A. Michaelides and E. Wang, Phys. Chem. Chem. Phys. 2013, 15, 6344.

37 See the supporting information in Ref. 2.

38 L. Zhang, Y. Wang, X. Zhang and Y. Ma, Phys. Rev. B, 2010, 82, 014108. 